\title{
Effect of Initial Geometry on Surface Flatness after Arc Welding Analyzed with MPS Method
}

\author{
Shigeki Hirasawa, Sadanori Toda, Tsuyoshi Kawanami and Katsuaki Shirai \\ Department of Mechanical Engineering, Kobe University, Kobe 657-8501, Japan
}

Received: December 25, 2014 / Accepted: January 14, 2015 / Published: February 25, 2015.

\begin{abstract}
The flow behavior in a typical arc welding process is very complex and features rapid melting and solidification. In this work, the flow in the melted region was determined by the effect of surface tension, viscosity, and gravity. Two-dimensional change of the geometry of the melted region was analyzed using the MPS (moving particle semi-implicit) method to melt a small upper plate in a hole at the center of a base plate. The relation between the initial geometry of the upper plate and the surface flatness after welding was calculated. The calculation results showed that the upper plate with a triangular notch at its center minimized the surface unevenness after welding. The depth of notch and thickness of the upper plate were optimized.
\end{abstract}

Key words: Melting, surface tension, flow, particle method, numerical analysis, optimization.

\section{Introduction}

In order to enable more precise manufacturing technology, it is indispensable to develop the CAE (computer aided engineering) technology to determine the optimal manufacturing condition and optimal process-control method. Welding is the basic manufacturing technology in mechanical engineering and there have thus been many studies on the welding process. However, the welding process is accompanied by complex phenomena such as the rapid phase change of melting and solidification and the flow of melted liquid by surface tension, viscosity, and gravity. In practice, process conditions for new welding conditions are still being determined by trial-and-error experiments.

There have been a few previous works on welding process simulation. Ko et al. [1] reported a GTAW (gas tungsten arc welding) process simulation using the SOLA-VOF (solution algorithm for transient fluid flow and volume of fluid) method to calculate the

Corresponding author: Shigeki Hirasawa, professor, research fields: thermo fluid control and heat transfer in equipment. E-mail: hirasawa@kobe-u.ac.jp. effect of surface depression on the flow of melted liquid and geometry. They showed that simulation using the SOLA-VOF method could reduce the computation cost reasonably without reducing accuracy. Ito et al. [2] reported a TIG (tungsten inert gas) welding process simulation using the $\mathrm{SPH}$ (smoothed particle hydrodynamics) method. Kanemaru et al. [3] reported a TIG-MIG (metal inert gas) hybrid welding process simulation using the SIMPLER (semi-implicit method for pressure linked equations revised) method to calculate the temperature, velocity, electric potential, and current flow distributions. Kong et al. [4] reported a GTAW process simulation using the FEM (finite element method) including effects of surface tension gradient, buoyancy force, arc pressure, and arc drag force to drive the fluid flow. Sreeraj et al. [5] reported a GMAW (gas metal arc welding) process simulation using the simulated annealing algorithm. Hirasawa and Haneda [6] reported the change in profile caused by melting, flowing, and solidifying in the arc welding process by analysis with the FEM. We clarified the conditions casing unsatisfactory welding, including undercuts, humping beads, separated beads, and 
melt-down weld. Recently, Koshizuka [7] developed a MPS (moving particle semi-implicit) method good for simulation of flow with a free surface. The explicit MPS method has the benefit of easy parallel computation using a GPU (graphic processing unit). Ochi et al. [8] reported a welding process simulation using a hybrid of the grid method and the explicit MPS method.

In this work, we clarified the optimum parameters to achieve surface flatness after arc welding of a small upper plate in a hole at the center of a base plate using a numerical simulation with the MPS method. The paper is organized as follows: Section 2 explains analytical model; Section 3 presents the calculation results; finally, Section 4 summarizes results.

\section{Analytical Model}

Fig. 1 shows the 2D calculation model of the arc welding of a small upper plate in a hole at the center of a base plate. The base plate was $0.8 \mathrm{~mm}$ thick and the width of the hole at the center was $2 \mathrm{~mm}$. The upper plate had a center thickness of $0.7 \mathrm{~mm}$ and a width of $3 \mathrm{~mm}$ and was placed on the hole before welding. Fig. 2 shows the ideal final structure after welding. Our goal was to achieve a flat upper surface after welding, as shown in Fig. 2. We calculated the change of geometry during the melting of the upper plate and the change of the flow by surface tension, viscosity, and gravity in the arc welding process.

The continuity equation and the momentum equation for a flow of fluid with a free surface are shown in Eqs. (1) and (2).

$$
\begin{gathered}
\frac{D \rho}{D t}=0 \\
\frac{D \boldsymbol{u}}{D t}=-\frac{1}{\rho} \nabla p+\nu \nabla^{2} \boldsymbol{u}+\boldsymbol{g}
\end{gathered}
$$

Here, $\boldsymbol{u}$ is velocity vector, $t$ is time, $p$ is pressure, $\rho$ is density, $v$ is dynamic viscosity, and $\boldsymbol{g}$ is gravity vector. The surface tension force was given on the free surface boundary. These equations were calculated using the MPS method [7]. The accuracy of our numerical simulation of flow with surface tension was confirmed by comparison with Lamb's analysis [9] for movement of a 2D liquid droplet.

The plates were aluminum, density was 2,350 $\mathrm{kg} \cdot \mathrm{m}^{-3}$, dynamic viscosity was $6.0 \times 10^{-7} \mathrm{~m}^{2} \cdot \mathrm{s}^{-1}$, and surface tension was assumed to be $0.1 \mathrm{~N} \cdot \mathrm{m}^{-1}$ for the oxide surface. Initial distance of particles was 0.05 $\mathrm{mm}$, total number of particles was 1,100 , and time step of calculation was $1.0 \times 10^{-7} \mathrm{~s}$. For simplicity, in our analysis, we did not calculate the temperature change and instead gave the condition of the time change of the melting region, which was obtained by our experimental results. The given condition of the time change of the melting region was that the initial expanding speed of the horizontal melting region in the upper plate was $0.2 \mathrm{~m} \cdot \mathrm{s}^{-1}$, the base plate near the hole melted at $0.0025 \mathrm{~s}$, and all of the upper plate melted at $0.0035 \mathrm{~s}$.

\section{Calculation Results}

Fig. 3 shows the calculation results of the change of geometry when the upper plate was a normal plate with no notches. At time $0.005 \mathrm{~s}$ (Fig. 3b), the upper part of the upper plate melted and started to gather to the center by the surface tension force. At time $0.013 \mathrm{~s}$ (Fig. 3c), the upper geometry gathered to the center and the melted part moved down by gravity. Under this condition, the upper surface geometry after welding was wavy and not flat.

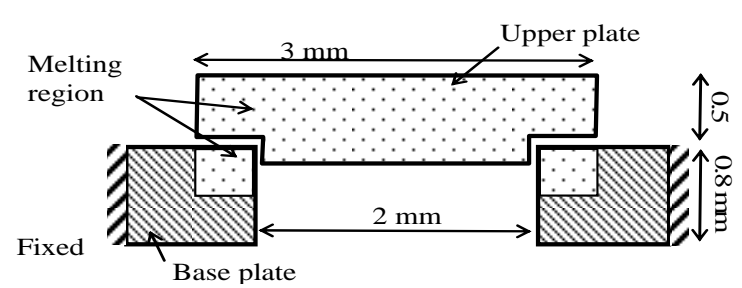

Fig. 1 Calculation model.

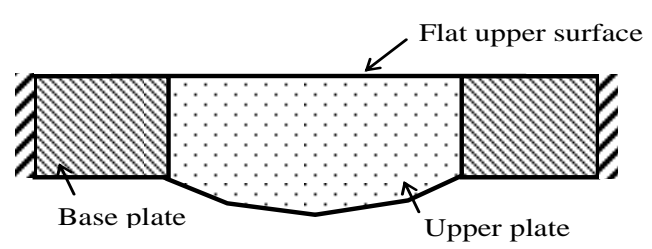

Fig. 2 Ideal structure after welding. 
Fig. 4 shows the calculation results of the change of geometry when the upper plate had a triangle notch of $0.2 \mathrm{~mm}$ in depth at the center. At time $0.005 \mathrm{~s}$ (Fig. 4b), the center of the upper part had a little dent, and at time $0.01 \mathrm{~s}$ (Fig. 4c), the upper surface came close to being flat compared with that in Fig. 3c. We defined the surface unevenness as the height of unevenness on the surface, as shown in Fig. 3c. Fig. 5 shows the calculation results of the relation between the surface unevenness and the depth of a triangular notch for the center thickness of the upper plate of 0.7 $\mathrm{mm}$. When the depth of the triangle notch was $0.2 \mathrm{~mm}$, the surface unevenness was the minimum. Fig. 6 shows the relation between time at minimum unevenness and the depth of a triangular notch.

We also calculated other geometries of the upper plate: with tapered edges, shown in Fig. 7, with

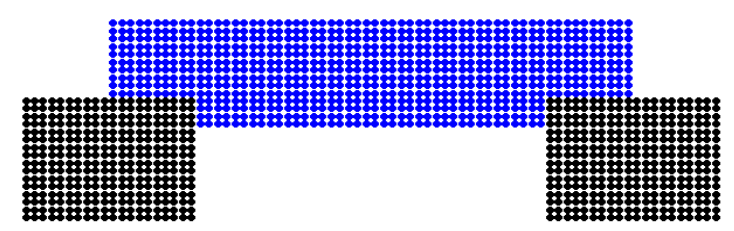

(a) At time $0.0006 \mathrm{~s}$

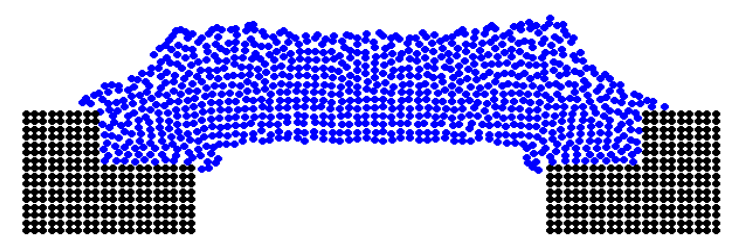

(b) At time $0.005 \mathrm{~s}$

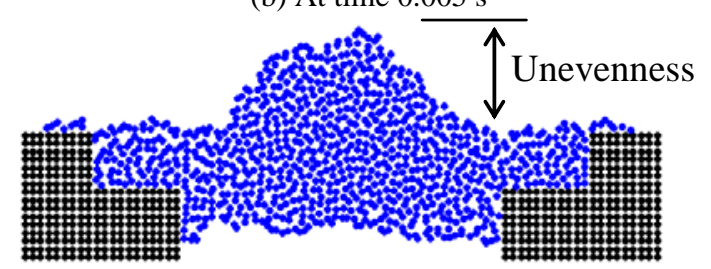

(c) At time $0.013 \mathrm{~s}$

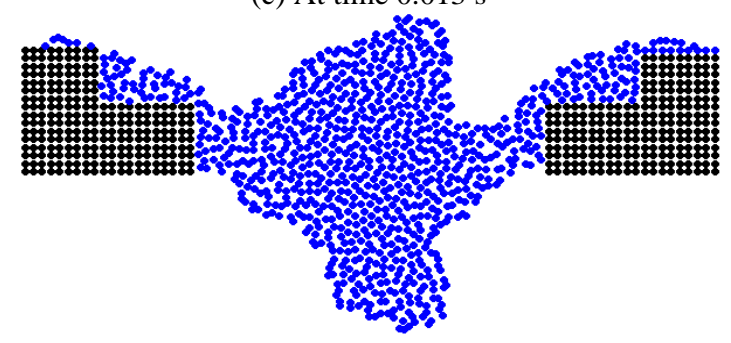

(d) At time $0.015 \mathrm{~s}$

Fig. 3 Normal upper plate.

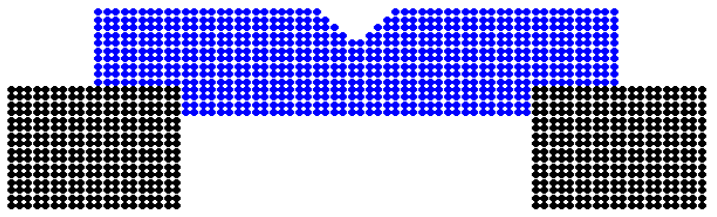

(a) At time $0.0006 \mathrm{~s}$

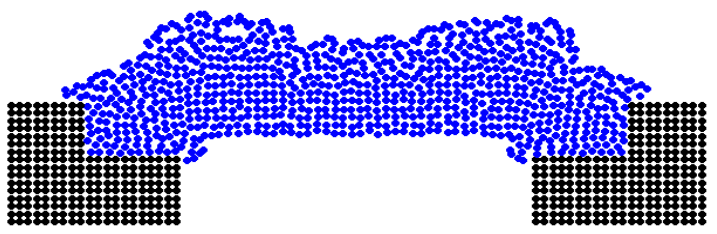

(b) At time $0.005 \mathrm{~s}$

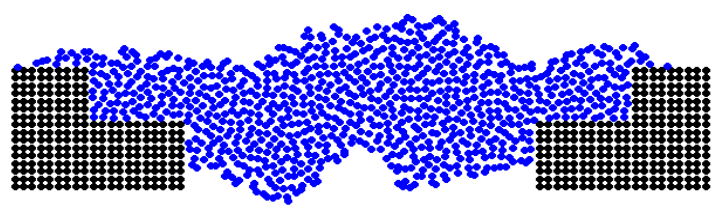

(c) At time $0.01 \mathrm{~s}$

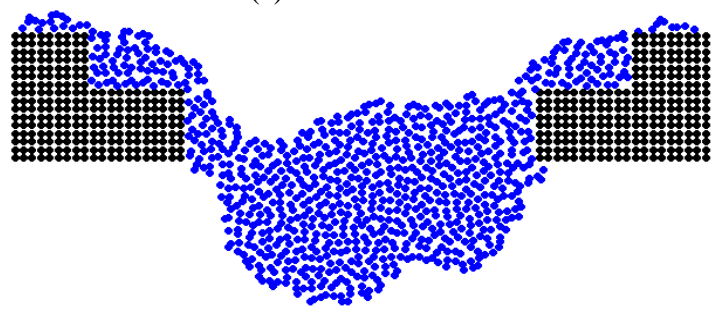

(d) At time $0.015 \mathrm{~s}$

Fig. 4 Upper plate with notch of $0.2 \mathrm{~mm}$ in depth.

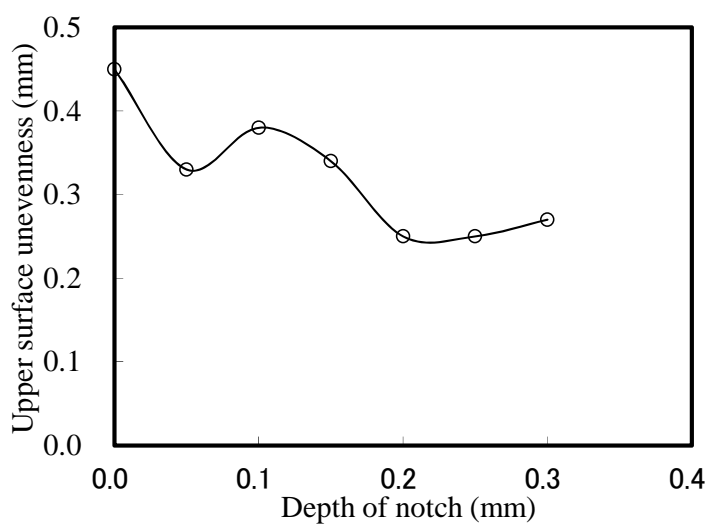

Fig. 5 Relation between surface unevenness and depth of triangular notch.

protruded edges, shown in Fig. 8, and with two notches, shown in Fig. 9. We found that the condition of the triangle notch of $0.2 \mathrm{~mm}$ in depth at the center (shown in Fig. 4) had the minimum upper surface unevenness after welding.

Fig. 10 shows the calculation results of the relation between the surface unevenness and the initial thickness 


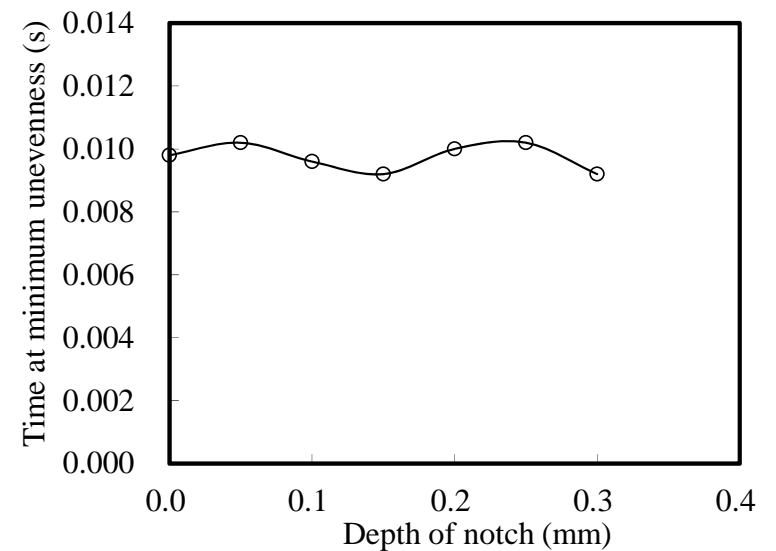

Fig. 6 Relation between time at minimum unevenness and depth of triangular notch.

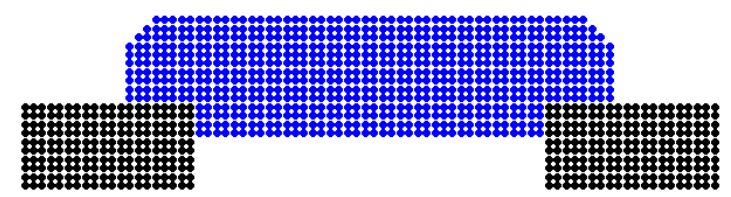

(a) Initial

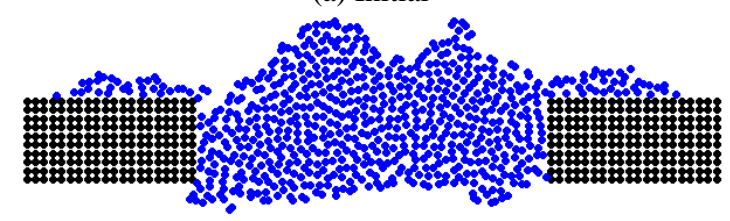

(b) At time $0.008 \mathrm{~s}$

Fig. 7 Upper plate with tapered edges.

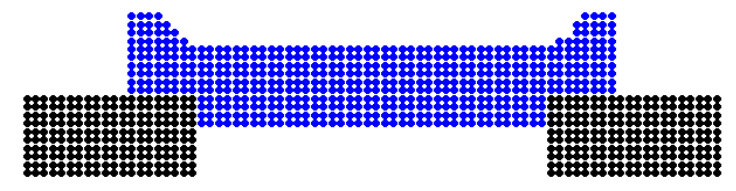

(a) Initial

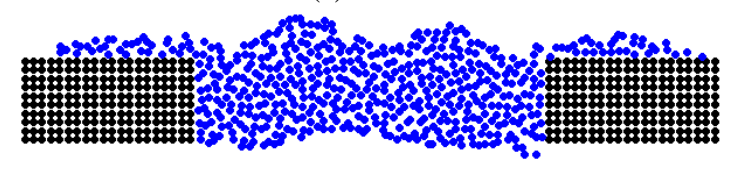

(b) At time $0.006 \mathrm{~s}$

Fig. 8 Upper plate with protruded edges.

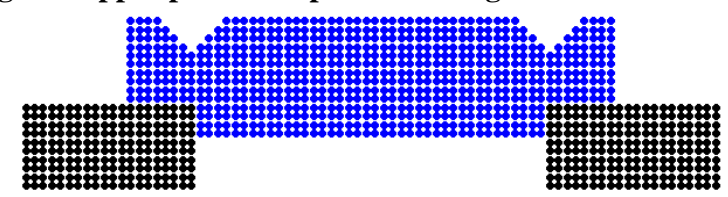

(a) Initial

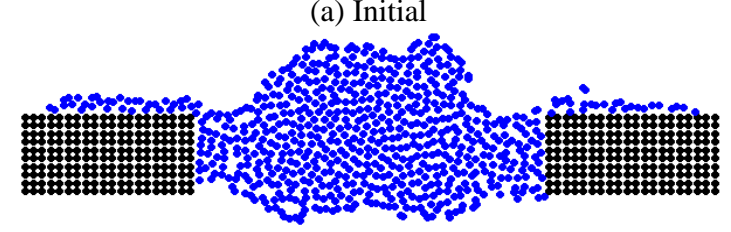

(b) At time $0.007 \mathrm{~s}$

Fig. 9 Upper plate with two notches.

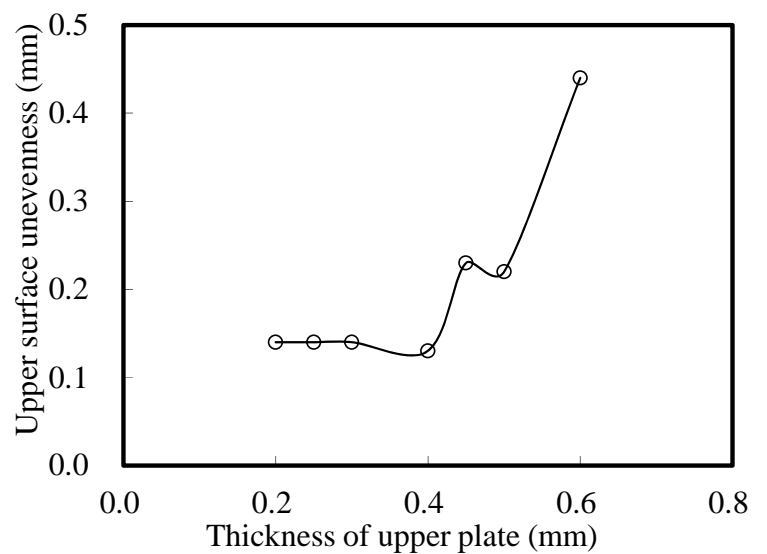

Fig. 10 Relation between surface unevenness and initial thickness of upper plate.

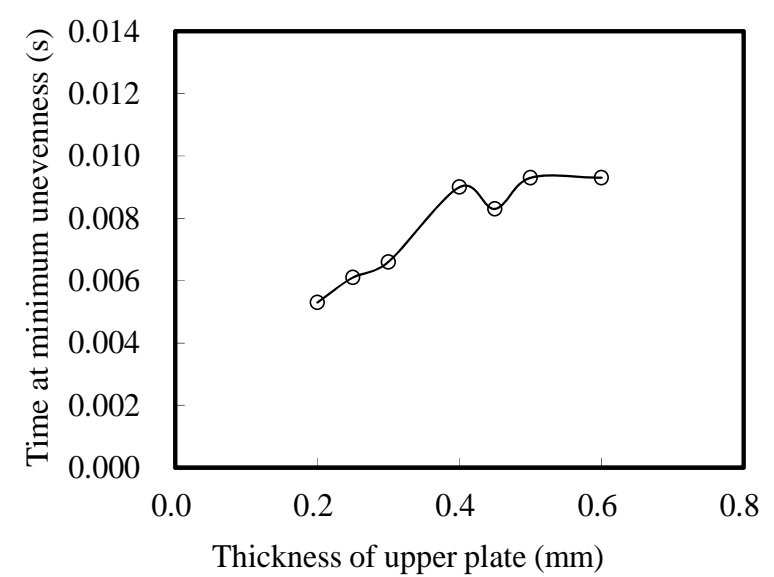

Fig. 11 Relation between time at minimum unevenness and initial thickness of upper plate.

of a normal upper plate with no notches. When the initial thickness of the upper plate was $0.4 \mathrm{~mm}$, the surface unevenness was the minimum. Fig. 11 shows the relation between time at minimum unevenness and the initial thickness of the upper plate.

\section{Conclusions}

We analytically examined the effect of initial geometry to achieve surface flatness after arc welding of a small upper plate in a hole at the center of a base plate. The following results were obtained:

(1) The surface unevenness after welding could be controlled via the initial geometry of the upper plate;

(2) The condition of the triangle notch of $0.2 \mathrm{~mm}$ in depth at the center of the upper plate had the minimum upper surface unevenness after welding. Also, the initial thickness of the upper plate of $0.4 \mathrm{~mm}$ with no 
notches had the minimum surface unevenness after welding.

\section{References}

[1] Ko, S. H., Choi, S. K., and Yoo, C. D. 2001. "Effects of Surface Depression on Pool Convection and Geometry in Stationary GTAW.” Welding Journal 80 (2): 39-45.

[2] Ito, M., Izawa, S., Fukunishi, Y., and Shigeta, M. 2012. "SPH Simulation of Gas Arc Welding Process." Presented at the 7th International Conference on Computational Fluid Dynamics, Hawaii, USA.

[3] Kanemaru, S., Sasaki, T., Sato, T., Mishima, H., Tashiro, S., and Tanaka, M. 2012. "3D Numerical Analysis Model for TIG-MIG Welding Process.” Quarterly of Japan Welding Society 30 (4): 306-12.

[4] Kong, K., Asserin, O., Gounand, S., Gilles, P., Bergheau, J. M., and Medale, M. 2012. "3D Finite Element Simulation of TIG Weld Pool.” Presented at the IOP
(Institute of Physics) Conf. Series: Materials Science and Engineering, Madrid, Spain.

[5] Sreeraj, P., Kannan, T., and Maji, S. 2013. "Prediction and Control of Weld Bead Geometry in Gas Metal Arc Welding Process Using Simulated Annealing Algorithm.” International Journal of Computational Engineering Research 33 (1): 213-22.

[6] Hirasawa, S., and Haneda, M. 2002. "Geometry Analysis in Arc Welding of Plates." In Proceedings of 12th International Heat Transfer Conference, 699-704.

[7] Koshizuka, S. 2005. Moving-Particle Semi-implicit Method. Tokyo: Maruzen Pub. Corporation.

[8] Ochi, N., Okawa, S., and Mochizuki, M. 2013. "Welding Process Simulation Using Hybrid Explicit MPS and Grid Method." Transaction of Japan Society for Computational Engineering and Science, Paper No. 20130005.

[9] Lamb, H. 1945. Hydrodynamics. UK: Cambridge Univ. Press. 